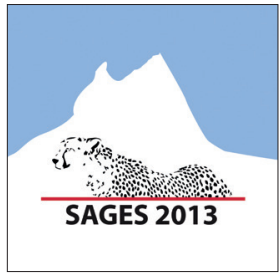

\title{
SAGES Congress
}

16 - 18 August 2013

Drakensberg, South Africa

SAGES abstracts

S Afr Med J 2013;103(8):568-575. DOI:10.7196/SAMJ.7178



\section{ORAL PRESENTATIONS}

Disease presentation and clinical course in black, coloured and white Crohn's disease patients in the Western Cape, South Africa

A Basson

University of the Western Cape, Cape Town, South Africa

Objective. To describe the clinical disease course of black, coloured and white Crohn's disease $(\mathrm{CD})$ patients from the gastrointestinal clinics of the Groote Schuur and Tygerberg hospitals.

Methods. Disease phenotype was described in consecutive CD patients diagnosed prior to 2006, using scheduled appointment lists from September 2011 to March 2013. Seven black, 152 coloured and 35 white cases were identified. Patients with history of tuberculosis or amoebiasis were excluded. Age of onset, disease location, disease behaviour, surgical intervention, presenting symptoms, extraintestinal manifestations (EIM), smoking, and family history of inflammatory bowel disease (IBD) was assessed.

Results. Of the black patients, 5 had ileocolonic (71\%), 1 had ileal (14\%), 1 had colonic (14\%) involvement, no family history of IBD was reported, and 6 were smokers at diagnosis. Perianal disease was present in all except 2 ileocolonic patients. At diagnosis, 43\% required surgical intervention and $71 \%$ had fistulising disease. All black cases had EIMs. Of the coloured patients, ileocolonic (56\%) and colonic (26\%) involvement was most common with 55\% requiring surgical intervention at diagnosis. Coloured patients presented with a younger median age of onset (26 years; interquartile range 18 $33)$, by approximately 4 years, with higher prevalence of multiple presenting symptoms, compared with white and black cases. Of the white patients, ileal (43\%) and ileocolonic (40\%) involvement were most common and $48 \%$ required surgical intervention at diagnosis. Conclusions. Preliminary analysis suggests that ileocolonic involvement is most common between races; however, coloured patients have the highest proportion of surgical intervention at diagnosis and appear to be diagnosed younger, with more presenting symptoms.

\section{Prediction of treatment outcomes from manometric subtypes in achalasia patients S Botha, S Hlatshwayo, P Bornman, S Thomson, $\underline{S \text { Gabriel, }}$ G Watermeyer \\ University of Cape Town, Cape Town, South Africa}

Background. There is emerging evidence that suggests that response to achalasia therapy is influenced by the manometric subtype obtained by high-resolution manometry (HRM).

Objective. To evaluate the usefulness of manometric subtypes in predicting outcome of treatment.

Methods. HRM data were collected in all newly diagnosed achalasia patients prior to treatment with either pneumatic balloon dilatation (PBD) or laparoscopic Heller's myotomy (LHM). The manometric tracings were then classified into the 3 Chicago subtypes by an experienced physician. Dysphagia symptoms (recorded as Eckardt scores) were also documented before and after treatment.

Results. Nine of the 31 patients had achalasia type 1 (29\%), 21 had type $2(68 \%)$ and only 1 had type $3(3 \%)$. The median follow-up time was 8 months (interquartile range 5 - 15). The success rate was $67 \%$ in subtype 1, 76\% in subtype 2 and $100 \%$ in subtype 3 (only 1 patient). Overall, the success rate was $74 \%$ and the difference between PBD and LHM was not statistically significant (PBD 76\% v. LHM 67\%; $p=0.890$ ).

Conclusion. There is a pattern towards better outcome in those with type 2 manometric phenotype than those with type 1 . However, the patient numbers in this study were too small to make firm conclusions.

Endoscopic ultrasound in the drainage of symptomatic pancreatic and peri-pancreatic fluid collections

S Burmeister, D Levin, $S$ Thomson

University of Cape Town, Cape Town, South Africa

Background. Endoscopic drainage has been shown to be a safe and effective means of draining symptomatic pancreatic and peripancreatic fluid collections occurring as a result of both acute and chronic pancreatitis. Its use, however, may be limited by both technical and patient-related factors. The development of endoscopic ultrasound has been purported to facilitate the endoscopic drainage of such collections as well as reduce complications.

objectives. This single-centre prospective study evaluated our initial experience with this modality in the management of these collections. Methods. Between February 2011 and May 2013, 31 patients (18 men, 13 women; mean age 42.2 years; range 11 - 77) with symptomatic pancreatic or peri-pancreatic fluid collections, occurring as a complication of acute $(n=23)$ or chronic pancreatitis $(n=8)$, were considered for endoscopic drainage on the basis of findings on computed tomography (CT) or magnetic resonance imaging (MRI). Results. The pancreatic/peri-pancreatic fluid collection was successfully accessed in 31 patients (100\%). Twenty-three (74\%) patients underwent placement of 1 or more plastic pigtail stents, 3 (10\%) had placement of a removable, covered self-expanding metal stent, $3(10 \%)$ were managed with aspiration only and $2(6 \%)$ patients could not be endoscopically drained and proceeded to surgical drainage, post endoscopic procedure. Three patients developed delayed complications relating to endoscopic drainage and required surgical intervention: development of sepsis within a residual collection $(n=1)$, delayed gastrointestinal perforation related to an endoscopically placed plastic stent $(n=1)$ and migration of a self-expanding metal stent into a partially drained cavity $(n=1)$. Endoscopic management was successful in $26(84 \%)$ patients. Two patients developed pneumoperitoneums following endoscopic intervention, but were successfully managed 
conservatively with bowel rest and antibiotics. There were no instances of early, clinically significant bleeding or gastrointestinal perforation related to the procedure.

Conclusion. Endoscopic ultrasound facilitates safe and effective, minimally invasive management of pancreatic and peri-pancreatic fluid collections occurring as a result of either acute or chronic pancreatitis.

The sero-prevalence of hepatitis C infection in an HIVinfected male population of heterosexual and men who have sex with men (MSM) in Cape Town

$\underline{\text { N Gogela }},{ }^{1,2}$ M Sonderup, ${ }^{1}$ K Rebe, ${ }^{3}$ W Spearman

${ }^{1}$ Division of Hepatology, University of Cape Town and Groote Schuur Hospital, Cape Town, South Africa

${ }^{2}$ GIT Clinic, University of Cape Town and Groote Schuur Hospital, Cape Town, South Africa

${ }^{3}$ Health4Men Clinic, Anova Health Institute, Cape Town, South Africa

Background. Hepatitis C virus (HCV) is a significant health problem with over $3 \%$ of the world's population infected. Transmission is mostly through parenteral routes via needles or exposure to blood products pre-1990. Heterosexual transmission is uncommon, but men who have sex with men (MSM) have an elevated risk of acquiring both HIV and HCV. The estimated local prevalence of $\mathrm{HCV}$ is $0.3 \%-1 \%$ while in Western Cape blood donors it is $<0.3 \%$. Co-infection rates among MSM who are injecting drug users in Europe and the USA are high; however, local prevalence is unknown. Methods. A prospective study was initiated and confirmed HIVpositive patients were recruited from various hospitals and clinics around Cape Town, including a dedicated MSM clinic. All patients were $\geq 18$ years old and those who consented completed a confidential questionnaire surveying risk stratification, sexual orientation, blood transfusion pre-1990 and drug use. All were screened for HCV.

Results. To date, 168 male patients have been recruited, 51\% ( $n=86)$ MSM and $49 \%(n=82)$ heterosexual (mean age 38.9, standard deviation (SD) \pm 7.9 years and $39.0 \pm 7.8$ years). Ethnically, in the MSM and heterosexual group $75 \%$ v. $15 \%$ were white; $16 \%$ v. $77 \%$ were black and $8 \%$ v. $7 \%$ were of mixed ancestry. Overall $6 \%(n=10)$ tested HCV antibody positive, all from the MSM cohort, representing $12 \%$ of this group. All HCV-positive patients had a history of prior or current drug use.

Conclusion. These interim results suggest that our local HCV prevalence in HIV-positive patients is underestimated, risk seems to be limited to the MSM sub-group. Targeted routine HCV testing is this group may be warranted.

Oesophageal cancer in Pietermaritzburg: The shocking timeline to diagnosis and definitive management M Govender $^{1,2}$

${ }^{1}$ Nelson Mandela School of Medicine, University of KwaZulu-Natal, Durban, South Africa

${ }^{2}$ Greys Hospital, Pietermaritzburg, South Africa

Background. Despite advances in management, oesophageal cancer remains a disease with dismal outcomes. Most patients present with advanced lesions amenable only to palliation.

objective. To quantify the oesophageal cancer timeline, from first symptom to definitive management, and to identify undue delays and points of potential impact.

Methods. This was an audit of patients seen with oesophageal cancer at Greys Hospital in Pietermaritzburg from January to
November 2012. Patients were identified from records at oncology and surgical clinics and the gastrointestinal unit. A retrospective analysis of records was performed and a timeline plotted, which included duration of symptoms prior to first presentation, and time to diagnosis and to definitive management.

Results. A total of 113 patient charts were reviewed. The time to first visit ranged from immediate presentation to a 12-month delay, with the average symptom duration being 5 months. Establishing a diagnosis took an average of 1 month from first presentation but varied from 0 to 8 months. Once a patient was diagnosed, definitive management was instituted on average 1 month later (range 0 7 months). The overall timeline from first symptom to definitive management was 2 - 14 months (mean 7).

Conclusion. There are unacceptably long delays in the presentation, diagnosis and management of oesophageal cancer in our environment. The problem is multifaceted. Patient education and improved referral systems may result in earlier presentation. The use of protocols and a multidisciplinary team may facilitate rapid diagnosis and management. Only by significantly reducing delays can we hope to affect outcomes.

\section{Pneumatic balloon dilatation v. laparoscopic Heller myotomy: A teaching hospital experience}

S Hlatshwayo, S Botha, P Bornman, $S$ Thomson, G Chinnery,

G Watermeyer, D Levin

University of Cape Town, Cape Town, South Africa

Background. Even though the European Achalasia Trial showed that pneumatic balloon dilatation (PBD) was as good as laparoscopic Heller myotomy (LHM) in primary achalasia, many healthcare professionals still consider LHM to be superior to PBD.

Objective. To compare the two treatment modalities in a cohort of patients treated in a teaching hospital.

Methods. Prospectively collected data from newly diagnosed achalasia patients managed by LHM and PBD were analysed. Symptoms of achalasia were documented using Eckardt scores (derived from the symptoms of weight loss, dysphagia, chest pain and regurgitation). The primary outcome was effective relief of symptoms, defined as an Eckardt score $<3$ and/or the need for alternative treatment. The secondary outcome was the complication rate in the two arms.

Results. Seventy-two of a total of 112 patients (64\%) had PBD while the remaining 40 (36\%) had LHM. The median follow-up period was 34 months (range 2 -116). Therapeutic success rate in the LHM arm was $75 \%(30 / 40)$ v. $67 \%(48 / 72)$ in the PBD arm. The differences between the two treatment modalities were not statistically different $(p=0.717)$. There were 2 perforations $(3 \%)$ following PBD and 2 patients (5\%) had mucosal breach during LHM. There were no deaths related to the procedures. One patient had aspiration pneumonia and another had minor wound sepsis in the LHM group. Conclusion. Pneumatic balloon dilatation was found to be equivalent in efficacy to LHM in patients with newly diagnosed achalasia. The complication rate was low in both groups.

\section{Comparison of the diagnostic yield and clinical outcomes of an approach based on initial double balloon enteroscopy v. initial capsule endoscopy in patients with obscure gastrointestinal bleeding E Kamau, $S$ Thomson}

University of Cape Town, Western Cape, South Africa 
Objective. To describe the diagnostic yield and clinical outcomes of a strategy based on initial capsule endoscopy followed by double balloon enteroscopy v. the use of double balloon enteroscopy alone in the management of obscure gastrointestinal bleeding.

Methods. This was a retrospective descriptive study of the medical records of 26 patients who had double balloon enteroscopy alone and 35 patients with initial capsule endoscopy followed by double balloon enteroscopy at the Groote Schuur Hospital between May 2009 and April 2013.

Results. Occult gastrointestinal bleeding was the primary indication for subsequent procedure in $81 \%$ of patients who underwent double balloon enteroscopy alone and in $51 \%$ of the patients who had initial capsule endoscopy. Of patients with double balloon enteroscopy alone, $61.5 \%(n=16)$ had positive findings. The small bowel findings included angiodysplasia $(n=6)$, tumours $(n=3)$ and ulcers $(n=4)$. Argon plasma photocoagulation was successfully applied in all patients with angiodysplasia and biopsies taken in the patients with tumours. In the group who had initial capsule endoscopy, the diagnostic yield was $77.1 \%(n=27)$ with capsule endoscopy and $71.4 \%(n=25)$ with double balloon enteroscopy $(p=0.157) ; 74.6 \%$ of the findings correlated positively. The most common finding was angiodysplasia and argon plasma coagulation (APC) was applied successfully in all cases. The clinical outcomes at 6 months were favourable in $46.1 \%$ and $60 \%$ of patients with double balloon enteroscopy alone and initial capsule enteroscopy, respectively ( $p=0.016$ ).

Conclusion. Capsule endoscopy improved the diagnostic yield of double balloon enteroscopy by $10 \%$ and this resulted in significantly better short-term clinical outcomes.

A prospective review of the endoscopic management of large bile duct calculi using controlled radial expansion (CRE) balloon dilatation v. sphincterotomy

E Loots, S Rambarran, F Anderson

Inkosi Albert Luthuli Central Hospital, University of KwaZulu-Natal,

Durban, South Africa

Background. Choledocholithiasis can present with varying sizes, number of calculi and associated complications. Endoscopic techniques allow for early interventions and less invasive surgery.

Objective. This study examines the techniques used in the management of large bile duct calculi.

Methods. All patients presenting for an endoscopic retrograde cholangiopancreatography (ERCP) were prospectively examined from June 2011 to May 2013 in a single unit. Information was collected where bile duct calculi were $>9 \mathrm{~mm}$. Demographic information, haematological results and radiological investigations were collected. The type of endoscopic intervention, outcome and subsequent management were documented. Comparison was made between the sphincterotomy group and controlled radial expansion (CRE) balloon dilatation group. The $\chi^{2}$ test was used for categorical data and the Student's $t$-test for continuous data in the statistical analysis.

Results. A total of 270 ERCPs were performed between June 2011 and May 2013. Forty-five patients were identified, comprising 13 males and 33 females. Five patients had prior magnetic resonance cholangiography (MRCP) and 2 had endoscopic ultrasound. Fifty-two ERCP procedures were performed. Twenty-seven patients had CRE dilatation and 18 sphincterotomy. There was a statistical difference between albumin and alkaline phosphatase (ALP) levels, but it was not noted with bilirubin, $\gamma$-glutamyltransferase (GGT), alanine transaminase (ALT), haemoglobin and international normalised ratio (INR). The average number of calculi was 6 with an average size of $16 \mathrm{~mm}$. Endoscopic failure was noted in a third of patients. The sphincterotomy group had 3 complications: a pancreatitis, retroperitoneal perforation and a lodged Dormia basket. There were no procedure-related complications in the CRE group reaching statistical significance.

Conclusion. Endoscopic therapy is effective in the majority of large choledocholithiasis. The use of CRE balloon sphincterotomy greatly facilitates ductal clearance and reduces procedure-related complications. MRCP remains underutilised as a non-invasive investigation in a resource-constrained environment.

\section{Solitary extramedullary plasmacytoma of the colon, rectum and anus: $A$ collective review $\underline{\text { M Mjolii }}{ }^{1,2}$ N Vorajee, ${ }^{1}$ J Howlett ${ }^{1,2}$ T Madiba ${ }^{1}$ \\ ${ }^{1}$ Pietermaritzburg Hospital Complex, Pietermaritzburg, South Africa \\ ${ }^{2}$ University of KwaZulu-Natal, Durban, South Africa}

Objective. To determine treatment characteristics and clinical outcomes of patients with solitary extramedullary plasmacytoma (SEP) of the colon, rectum and anus.

Methods. A systematic literature search was performed to identify all case reports and series on SEP of the colon, rectum and anus. A single case treated at our institution was added to these case reports. All multiple myeloma cases were excluded. Patient characteristics, treatment, and outcomes of each patient were entered in a database and a pooled analysis was carried out.

Results. Twenty-eight patients were identified with a mean age of 53.5 years. Males were more common, with a male:female ratio of 3:1. The common symptoms were pain $(n=14)$, rectal bleeding $(n=8)$, and constitutional symptoms $(n=7)$. The most common sites of colonic involvement were sigmoid colon $(n=9)$, caecum $(n=8)$ and rectum $(n=5)$. The most common surgical intervention was segmental colectomy. Treatment was more variable where the rectum was involved and consisted of endoscopic resection, radiotherapy and rectal resection. Twenty-six patients had a curative resection with 2 locoregional recurrences. Follow-up was documented in 18/28 patients. The mean duration of follow-up was 13.9 months.

Conclusion. Solitary extramedullary plasmacytoma is rare and commonly affects the caecum and sigmoid colon. No conclusions can be drawn regarding the optimal management and their outcomes. However, this review may help the surgeon with the available management options to consider.

\section{The impact of biological therapy in patients with inflammatory bowel disease at Groote Schuur Hospital A Rajula, $S$ Thomson, G Watermeyer}

University Of Cape Town, Cape Town, South Africa

Objectives. To audit the use of biologicals in patients with inflammatory bowel disease (IBD).

Methods. A retrospective analysis of IBD patients treated with biologicals (from 2009 to 2013) was performed. Relevant data pertaining to demographics, disease characteristics, concomitant medications and response were entered into a database and analysed.

Results. A total of 24 patients were available for analysis (mean age at diagnosis 28.6 years, standard deviation (SD) \pm 12.2 ), $67 \%$ were male. The median duration of disease prior to biologicals was 66 months (interquartile range $20-120$ ). The majority were of mixed ancestry (54\%) and 59\% were smokers. There were only 4 patients with ulcerative colitis (UC), all treated with infliximab for acute, severe UC. All responded well and avoided colectomy. Of the cohort (20/24), 
$83 \%$ had Crohn's disease (CD); of these $25 \%$ had ileocaecal disease, $30 \%$ isolated colonic CD and $45 \%$ ileocolonic CD. Only 20\% (4/20) had inflammatory (non-fistulising, non-penetrating $\mathrm{CD}$ ) at the time of biological initiation, while $15 \%$ had a stricturing phenotype and $65 \%$ penetrating CD; $45 \%$ had perianal disease. Infliximab (IFX) and adalumimab were given in $55 \%$ and $45 \%$, respectively, and $95 \%$ were receiving concomitant immunomodulators (19/20), 17 of these were receiving azathioprine. Only $40 \%$ of patients with $\mathrm{CD}$ had a primary response. None of the baseline variables significantly predicted this outcome on univariate analysis.

Conclusion. While IFX is of value in UC as colon salvage therapy, we report disappointing primary response rate in our cohort with $\mathrm{CD}$. This likely reflects the introduction of these agents in advanced complicated $\mathrm{CD}$ when irreversible damage has already occurred. While none of the baseline variables significantly predicted primary response, our sample size is likely too small for meaningful analysis.

\section{Triad of dysregulated signalling in human hepatocellular carcinoma suggests a multipronged approach is needed for therapeutic targeting M Setshedi, ${ }^{1,2}$ L Longato, ${ }^{2} \mathrm{~K}$ Ripp, ${ }^{2} \mathrm{~W}$ Chen, ${ }^{2}$ Q-G Nguyen, ${ }^{2} \mathrm{~J}$ Wands, ${ }^{2}$ S de la Monte ${ }^{2}$ \\ ${ }^{1}$ University of Cape Town, Cape Town, South Africa \\ ${ }^{2}$ Warren Alpert Medical School, Brown University, Providence, USA}

Background. Previous studies highlighted potential roles of aberrant activation of insulin/insulin receptor substrate (IRS), canonical Wnt/ $\beta$ catenin, or Notch/Jagged/aspartyl-(asparaginyl)- $\beta$-hydroxylase (AAH) as mediators of hepatocellular carcinoma (HCC) pathogenesis or progression. In addition, a role for pro-inflammatory mediators of cell injury, turnover, and DNA damage is under investigation in HCC development.

Objective. We examined the extent to which each of these pathways were upregulated in HCC v. peri-tumour liver tissue, and evaluated the potential contributions of pro-inflammatory cytokines as mediators of progressive liver injury.

Methods. Paired surgically resected HCCs $(n=8)$ and tumour-free periHCC livers $(n=8)$ were used to measure gene expression by quantitative reverse transcription-polymerase chain reaction (qRT-PCR), and immunoreactivity by either multiplex or duplex enzyme-linked immunosorbent assays (ELISAs).

Results. Insulin: HCC was associated with significantly upregulated expression of the insulin-like growth factor (IGF) 2 receptor, insulin receptor substrate (IRS) 1, IRS2, and IRS4 genes, increased signalling through IGF-1R $(p=0.001)$ and IRS1 $(p=0.015)$, and inhibition of glycogen synthase kinase (GSK)-3 $\beta$ activity relative to peri-tumour liver tissue. Wnt $/ \beta$-catenin: HCCs had significantly increased mRNA expression of Wnt $3(p<0.0001)$, Wnt5A ( $p=0.004)$, and Wnt5B ( $p=0.01)$ ligands, and frizzled- 6 receptor $(p=0.02)$, and decreased expression of secreted frizzled related protein (FRP) $1(p=0.01)$ and sFRP5 $(p=0.008)$ relative to peri-tumour tissues. Notch: HCCs had significantly increased expression of AAH, Humbug, Notch-1, Jagged-1, and hairy and enhancer of split-1 (HES-1) relative to peri-tumour tissues. Inflammation: multiplex ELISAs used to measure 24 different cytokines/chemokines, demonstrated increased expression of only interferon (IFN)- $\gamma$, and significantly reduced levels of interleukin (IL)- $1 \beta$, IL- 6 , tumour necrosis factor (TNF)-related apoptosis-inducing ligand (TRAIL), MIP-1 and $\beta$-nerve growth factor (NGF) in HCC relative to peri-tumour liver tissue. Conclusions. HCCs are associated with simultaneous dysregulation of 3 major signalling pathways that likely cross-talk through various mechanisms, including GSK-3 $\beta$. The prominent down-regulation of several pro-inflammatory cytokines suggests that compromised host surveillance mechanisms rather than inflammation-mediated cell injury may contribute to the pathogenesis or progression of HCC. Altogether, the data suggest that effective therapeutic targeting of HCCs will require multipronged approaches that abrogate dysregulation of the insulin/IRS, Wnt/ $\beta$-catenin, and Notch pathways and fortify host immune surveillance mechanisms.

\section{Characteristics of hepatitis B-associated hepatocellular carcinoma (HCC) in Cape Town}

M Sonderup, M Setshedi, W Spearman

Division of Hepatology, University of Cape Town and Groote Schuur Hospital, Cape Town, South Africa

Objective. Globally, hepatocellular carcinoma (HCC) is the 6th most prevalent cancer with $75 \%$ occurring in Southeast Asia and subSaharan Africa due to the endemicity of hepatitis B virus (HBV).

Methods. Prospectively since 2011, patients presenting to Groote Schuur Hospital with HCC where HBV was assessed as being the likely risk factor, were recruited. Patients were excluded if they had hepatitis $\mathrm{C}$ co-infection or alcohol as a dominant risk factor. Patient demographics, HBV characteristics, HCC features and other relevant data were recorded.

Results. Thirty-three patients - 52\% $(n=17)$ black, 36\% $(n=12)$ mixed ancestry and $12 \%(n=4)$ white - were evaluated (mean age 43.2 , standard deviation (SD) \pm 11.9 years). Males predominated $(85 \%, n=27)$ and $15 \%(n=5)$ were HIV co-infected, median CD $4^{+}$ count was 250 cells $/ \mu$ l (range 216 - 567). Five patients were HBsAgnegative but heterogeneous hepatitis B (HB) core IgG-positive, 1 having detectable HBV DNA. Of those HBsAg-positive, 32\% $(n=9)$ were $\mathrm{HBeAg}$-positive, 68\% ( $n=19) \mathrm{HBeAg-negative,} \mathrm{with}$ mean \pm SD HBV viral loads of $6.2 \pm 1.5$ and $4.5 \pm 2.3 \mathrm{log}$ copies $/ \mathrm{ml}$, respectively $(p=0.03)$. The median $\alpha$-fetoprotein $(\alpha-\mathrm{FP})$ was 4091 $\mu \mathrm{g} / \mathrm{l}$ (range 2.9 - 401986 ) with only $12 \%(n=4)$ having normal a-FP levels. In terms of liver function at presentation, median total bilirubin, albumin and international normalised ratio (INR) were $22 \mu \mathrm{mol} / \mathrm{l}$ (range 6 - 300), $29 \mathrm{~g} / \mathrm{l}$ (range 16 - $48 \mathrm{~g} / \mathrm{l}$ ) and 1.32 (range 0.99 -3.17); respectively. Interestingly, median alanine transaminase $(\mathrm{ALT})(61 \mathrm{U} / \mathrm{l}$; range 22 - 378) and aspartate transaminase (AST) (118 IU/l; range 23 - 1156) differed significantly $(p=0.0009)$. The majority, $64 \%(n=21)$, had multicentric HCC, $27 \%(n=9)$ with focal and $9 \%(n=3)$ with diffusely infiltrating tumour. Histologically $(n=16), 75 \%(n=12)$ had established cirrhosis while the majority, $56 \%(n=9)$ had poorly differentiated HCC. To date median survival is 4 months (range 1 - 24).

Conclusion. HBV-associated HCC continues to carry a poor prognosis with patients presenting late with essentially incurable disease. $a-F P$ remains a useful tumour marker.

\section{Randomised clinical trial: Effect of Lactobacillus plantarum 299v on symptoms of irritable bowel syndrome $\underline{\text { C Stevenson }},{ }^{1}{ }^{1}$ Blaauw, ${ }^{1}$ E Fredericks, ${ }^{2}$ J Visser, ${ }^{1}$ S Roux ${ }^{2}$ \\ ${ }^{1}$ Stellenbosch University, Cape Town, South Africa \\ ${ }^{2}$ Nelson Mandela Metropolitan University, Port Elizabeth, South Africa}

Background. Irritable bowel syndrome (IBS) is the most common diagnosis in gastroenterology. Its aetiology is unknown and therapeutic options are limited. Consensus guidelines and results from systematic reviews suggest that probiotics may be beneficial, although controversy still exists. 
Objective. To assess the symptomatic efficacy of Lactobacillus plantarum $299 \mathrm{v}$ for the relief of abdominal pain in patients with IBS fulfilling Rome II Criteria.

Methods. In this double-blind, placebo-controlled, paralleldesigned trial participants were randomised to receive daily either 2 capsules of L. plantarum 299v or a placebo for 8 weeks. Severity of abdominal pain was assessed with a visual analogue scale (VAS) at each visit and a quality-of-life IBS (QoL-IBS) questionnaire was also completed.

Results. Eighty-one participants were randomised. There was no significant difference in abdominal pain relief between the study and placebo groups $(p=0.800)$. There was also no difference in QoL-IBS scores between the groups $(p=0.687)$. Both groups had a significant improvement in abdominal pain scores from an average of 251.55 to $197.90(p<0.0001)$ over the study period, indicating a large placebo effect. Conclusion. An 8-week treatment with L. plantarum 299v did not provide symptomatic relief, particularly of abdominal pain and bloating, in patients fulfilling the Rome II Criteria.

\section{Predicting poor-outcome Crohn's Disease at the time of first diagnosis \\ G Watermeyer, S Thomson, L Myer \\ University of Cape Town, Cape Town, South Africa}

Background. Most patients with Crohn's disease (CD) develop complications such as strictures or fistulas over time, resulting in hospitalisation and surgery. Timely therapy with immunomodulators and biologicals may alter this natural history but carries a risk of sideeffects.

Objective. To identify predictive factors at diagnosis for poor-outcome $\mathrm{CD}$ to identify those who would most benefit from early, aggressive medical therapies.

Methods. A retrospective analysis of $120 \mathrm{CD}$ patients with followup exceeding 5 years was performed using two different definitions of poor-outcome $\mathrm{CD}$ : disabling $\mathrm{CD}$ within 5 years of diagnosis (DCD) and severe CD over the entire disease course (SCD). Clinical, demographic, laboratory and biological factors at diagnosis associated with these outcomes were evaluated by univariate and multivariate analysis.

Results. Within 5 years, 72\% (86/120) of patients developed DCD; on multivariate analysis, perianal disease (odd's ratio (OR) 11.0; 95\% confidence interval (CI) $1.1-94.7 ; p=0.03$ ) and ileal involvement (OR 5.4; 95\% CI 2.1 - 13.9; $p<0.001)$ were predictive. Overall $38 \%(45 / 120)$ developed SCD and on multivariate survival analysis penetrating behaviour (hazard ratio (HR) 2.9; 95\% CI 1.2 - 7.0; $p=0.02$ ), stricturing behaviour (HR 4.6, 95\% CI 1.5 - 14.6; $p=0.01$ ), perianal disease (HR 3.4; 95\% CI $1.6-7.2 ; p=0.01$ ) and biopsy granulomas (HR 2.4; 95\% CI $1.3-4.6$; $p=0.01$ ) were independently associated with time to development of SCD. When analysis was restricted to non-stricturing/non-penetrating disease, perianal $\mathrm{CD}$ and tissue granulomas remained strongly associated with $\mathrm{SCD}$ with a specificity of $99 \%$ and a positive predictive value of $89 \%$.

Conclusion. At diagnosis, factors predictive of subsequent SCD in our referral centre were stricturing or penetrating phenotype, perianal disease and tissue granulomas.

\section{POSTER PRESENTATIONS}

\section{Cold snare polypectomy for colonic polyps $>10 \mathrm{~mm}$

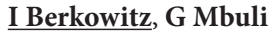 \\ Milpark Hospital, Johannesburg, South Africa}

Background. Cold polypectomy has been widely used for the removal of sub-centimetre colonic polyps. The technique appears to be both effective and safe and eliminates the risk of electrocautery. We report 3 cases of flat colonic polyps $>10 \mathrm{~mm}$, which were removed by cold snare polypectomy.

Methods. Flat colonic polyps $>10 \mathrm{~mm}$ were identified on colonoscopy in 3 patients. Two patients had polyps on the right side of the colon and 1 on the left side. The polyps were removed by cold snare polypectomy using an Exacto cold snare. One of the polyps was removed completely and the other 2 were removed piecemeal. The polyps were retrieved by suctioning into a snare trap, for histology.

Results. All 3 patients had their polyps successfully removed by cold snare polypectomy, with no complications, such as perforation or bleeding. Polyp removal was assessed to be complete with no residual polyp seen on careful endoscopic inspection.

Conclusion. Cold snare polypectomy appears to be both feasible and safe for the removal of polyps $>10 \mathrm{~mm}$ in size, either by complete or piecemeal removal.

\section{Bile duct perforation in an infant S Bisetty, M H Sheik Gafoor, V G Naidoo \\ University of KwaZulu-Natal, Durban, South Africa}

Background. Bile duct perforation can present with cholestasis. Less than 150 cases have been described worldwide and it is often misdiagnosed.
Case presentation. A 4-month-old infant was referred with problems of cholestasis and ascites. Biliary atresia was excluded. Histology from a laparoscopic liver biopsy was in keeping with a diagnosis of neonatal hepatitis. However, despite adequate therapy, the ascites worsened. The infant failed to thrive and was emaciated. Repeat liver biopsy was normal. The ascitic fluid was re-aspirated and bile stained fluid was drained. The plasma bilirubin levels were normal. A hepatobiliary iminodiacetic acid (HIDA) isotope scan demonstrated normal liver uptake and normal gall bladder filling, followed by extravasation of isotope from the extra-hepatic bile duct into the peritoneal space. Endoscopic retrograde cholangiography demonstrated a bile duct perforation. A stent could not be deployed across the defect and a portoenterostomy procedure was performed. Discussion. It is unclear whether the bile duct injury was due to spontaneous bile duct perforation (SBDP) or bile duct trauma from the initial liver biopsy. SBDP is the second most common surgical cause for cholestasis.

Conclusion. Bile duct perforation, if left undiagnosed and untreated, is potentially fatal. One needs to have a high index of suspicion, in correlation with the clinical signs and symptoms. A HIDA scan proved valuable in defining and localising the clinical problem.

Assessment of the critical flicker frequency measuring machine in diagnosis of minimal hepatic encephalopathy A Deshpande, R Borse

BJ Medical College, Pune, Maharashtra, India

Objectives. To assess the usefulness of a self-designed machine in measuring critical flicker frequency (CFF) for diagnosis of minimal 
hepatic encephalopathy (MHE), exploring cheaper alternatives for the diagnosis of MHE.

Methods. All patients with liver disease in Sassoon General Hospital in Pune, India over a period of 1 year were considered. Patients with liver disease but normal neurological examination participated in the study. Patients with overt hepatic encephalopathy (HE) or history of HE were excluded. Also patients with any overt neurological or ocular diseases were excluded. The number connection tests (NCT) $\mathrm{A}$ and $\mathrm{B}$ were considered standard for diagnosing MHE. All such patients were then exposed to the CFF measuring machine and readings were taken. Four such readings were obtained in one sitting and the average was calculated. Thirty-three patients fitting the criteria were diagnosed with MHE and considered for study. Most of the patients were encountered in the gastroenterology outpatients department (OPD) of the hospital. The control group $(n=35)$ consisted of subjects with similar educational status as patients, including relatives.

Results. There were 33 subjects with MHE (mean 43.67 years; male:female ratio 31:2). There was a negative correlation between the NCTs and CFF. The difference between the CFF values of the two groups was significant. Correlations between different tests were calculated by Spearman's rank-order correlation coefficient. A significance level of $p=0.05$ was used in all analyses.

Conclusion. This study concluded that the machine under consideration could effectively measure CFF and help in diagnosis of MHE in an OPD setting. The cost of development was US\$73.53 (Rs.4 000), which is affordable in various setups.

\section{Duodenal eosinophil count in patients with functional dyspepsia at Chris Hani Baragwanath Academic Hospital \\ M Hussein, R Ally, N Chopdat, W AbulHassan, B Bobat \\ University of the Witwatersrand, Johannesburg, South Africa}

Objective. To determine the duodenal eosinophil count in patients presenting with functional dyspepsia.

Methods. Patients presenting to the endoscopy unit for an upper gastrointestinal (GI) endoscopy were screened using a questionnaire and those who satisfied the Rome III Criteria for functional dyspepsia were included in the study. An upper GI endoscopy was done and patients who had any structural lesions were excluded. Recruitment continued consecutively until a sample size of 50 patients was attained. Three duodenal biopsies from the first part of the duodenum were taken to the laboratory to check for the total eosinophil count per 5 high power field (HPF).

Results. A total of 50 duodenal biopsies were done of which 3 had insufficient tissue sample for an eosinophil count and 7 were abnormal (i.e. had histopathological features of duodenitis). Forty biopsies were taken for the final analysis. The average age of the patients was 38 years and $85 \%$ were female. Thirty-two patients presented with early satiety, 27 with post-prandial bloating, 31 with abdominal fullness and 36 with epigastric pain syndrome. The mean duodenal eosinophil count was 35.6 (standard deviation $\pm 17.8)$ per $5 \mathrm{HPF} ; 0 \%$ had an above normal eosinophil count (>22 per $5 \mathrm{HPF}$ ).

Conclusion. Patients presenting with functional dyspepsia have a high duodenal eosinophil count. Randomised placebo-controlled clinical trials on anti-eosinophilic agents as treatment for functional dyspepsia need to be carried out.
Resistant Clostridium difficile infection and faecal transplantation in patients with inflammatory bowel disease at Wits Donald Gordon Medical Centre K Karlsson, P Barrow

Donald Gordon Medical Centre, University of the Witwatersrand, Johannesburg, South Africa

Objective. To describe our method and experience with faecal transplantation in treating patients with inflammatory bowel disease (IBD) and resistant Clostridium difficile infection.

Methods. We treated 3 patients - 1 inpatient due to disease severity and associated complications and 2 outpatients. Two failed both metronidazole and vancomycin, 1 failed metronidazole and was unable to purchase vancomycin. We chose the cheapest and easiest method of transplantation for our patients. This entailed commencement of a proton pump inhibitor (PPI) 24 hours pre-transplant, discontinuation of antibiotics the night before the procedure. Donors were willing to donate; history and examination confirmed no significant GI disease. Screening included: HIV; hepatitis B and C; stool for ova, cysts, parasites; culture; $C$. difficile polymerase chain reaction (PCR); and, in 1 donor, rota and adenoviruses. Donors were prescribed 1 sachet of Movicol for the day prior to donation and stool was kept cool during transportation. Stool was mixed using a liquidiser with sterile water until an acceptable consistency was reached and $50 \mathrm{ml}$ of the resulting solution was drawn into a syringe. A nasogastric (NG) tube was placed and the position checked by auscultation and aspiration of gastric juices. The stool solution was instilled via the NG tube. The NG tube was flushed with $50 \mathrm{ml}$ water and left in situ for $15 \mathrm{~min}$. The patient was kept for a further 15 - 30 min to ensure emesis did not occur. Stool was tested for C. difficile PCR at 4 weeks.

Results. All patients had a successful response with clearance of C. difficile and resolution of symptoms.

Conclusion. Faecal transplantation is a successful treatment option for recurrent $C$. difficile infection in patients with IBD.

\section{Adult nesidioblastosis: A problematic diagnosis and a treatment challenge \\ E Loots, F Anderson \\ Inkosi Albert Luthuli Central Hospital, University of KwaZulu-Natal, Durban, South Africa}

Background. Nesidioblastosisis an uncommon cause of hyperinsulinaemic hypoglycaemia and can significantly impact on quality of life. The condition refers to $\beta$-cell hyperplasia of the pancreas. The diagnosis is difficult to determine. Treatment requires medical and surgical therapies. Methods. A retrospective chart review was undertaken of all patients diagnosed with adult nesidioblastosis between January 2007 and May 2013. Biochemistry results, radiological reports, histological data and operative notes were collected and reviewed. Data were analysed using simple statistical methods.

Results. Four patients fulfilled the clinical and histological criteria for the diagnosis of nesidioblastosis. Medical therapy using optimal dosages of diazoxide failed to control symptoms in all of these patients rendering surgery necessary. The pertinent investigations and surgical outcomes are summarised in the table. Hypoglycaemia resolved in all patients after the initial resection. Three of four patients required further surgery after an average of 28 months for recurrence of hypoglycaemia.

Conclusion. The diagnosis of adult nesidioblastosis is difficult to make. Current localisation investigations proved unreliable. Medical 


\begin{tabular}{|c|c|c|c|c|}
\hline Patient & Localisation & LA & Initial resection & Outcome \\
\hline 1 & CST & + & $80 \% \mathrm{DP}$ & GI \\
\hline 2 & $\mathrm{EUS}+\mathrm{CST}$ & - & $70 \%$ DP, recurrence & $\begin{array}{l}95 \% \mathrm{DP} \\
\mathrm{DM}\end{array}$ \\
\hline 3 & $\mathrm{PET}+\mathrm{CST}$ & - & $40 \%$ DP, recurrence & $\begin{array}{l}80 \% \mathrm{DP} \\
\mathrm{DM}\end{array}$ \\
\hline 4 & CST & - & $40 \%$ DP, recurrence & $\begin{array}{l}80 \% \mathrm{DP} \\
\mathrm{DM}\end{array}$ \\
\hline
\end{tabular}

treatment with diazoxide has limited effect. Recurrent hypoglycaemia is not uncommon following initial pancreatic resection and diabetes mellitus after repeat surgery.

\section{Normal values of 24-hour ambulatory oesophageal multichannel intraluminal impedance-pH monitoring in a rural black African population \\ E J Ndebia, A Sammon, E Umapathy, J Iputo \\ Walter Sisulu University, Mthatha, South Africa}

Background. Impedance-pH monitoring is a new technique for the diagnosis of gastro-oesophageal reflux disease (GERD). This method detects liquid, mixed and gas reflux as well as its distribution, composition and clearing. There is no available data on oesophageal impedance monitoring in black African rural populations.

Objective. To define the normal pattern of gastro-oesophageal reflux of rural black Africans and compare them with similar studies.

Methods. Healthy volunteers were recruited and underwent 24-hour ambulatory multichannel intraluminal impedance-pH (MII-pH) monitoring. Gastro-oesophageal reflux episodes were detected and characterised by $\mathrm{pH}$ as acid, weakly acid or non-acid reflux and by composition as liquid and mixed reflux.

Results. Sixty-four participants were included in the study ( 16 males, 48 females). Mean age was 36 years (range 18 - 60). The median and the 95th percentile of the total reflux episodes was 37 and 82, respectively of which acidic, weakly acidic and non-acid reflux were median and 95th percentile 14 and 59, 12 and 46, and 2 and 20, respectively. The composition of the total reflux was 7 and 24 for liquid, 28 and 73 for mixed and 33 and 135 for gas reflux, respectively. The median bolus clearance was $16 \mathrm{~s}$ whereas acid was chemically cleared in $54 \mathrm{~s}$. More reflux was observed in the upright position while there was an increased alkaline reflux compared with similar Western studies.

Conclusion. This study demonstrates that it feasible to carry out an impedance-pH monitoring study in rural Africa, and that this provides normal values.

\section{'Downhill' oesophageal varices \\ S Onyango ${ }^{1,2}$ VG Naidoo, ${ }^{1}$ KA Newton ${ }^{1}$ \\ ${ }^{1}$ University of KwaZulu-Natal, Durban, South Africa \\ ${ }^{2}$ Kenyatta National Hospital, Nairobi, Kenya}

Background. 'Downhill' varices occur when superior vena cava (SVC) obstruction results in reversal of blood flow in the upper oesophageal venous plexus. 'Downhill' varices are usually asymptomatic with few cases of bleeding reported.
Case presentation. A 46-year-old Indian female on chronic haemodialysis was referred for endoscopy to evaluate persistent reflux symptoms. Physical examination revealed distended veins over the chest wall and a right subclavian haemodialysis catheter. There were no clinical features of cirrhosis and none of the traditional risk factors for cirrhosis were identified. Upper endoscopy showed a single variceal column in the proximal oesophagus with two short columns of varices at the distal oesophagus. No other endoscopic abnormalities were seen. Computed axial tomography with pulmonary angiography (CT-PA) showed a thrombus at the catheter tip in the SVC. Imaging also demonstrated a normal liver outline, normal portal vein and no significant splenomegaly. These findings led us to conclude that the varices were not due to portal hypertension ('uphill') but rather related to the SVC obstruction leading to the formation of 'downhill' varices.

Discussion. The incidental finding of varices in the absence of other features of portal hypertension should prompt suspicion of 'downhill' varices. Awareness of this condition may avoid unnecessary tests for liver disease. Bypassing the vascular obstruction is an additional therapeutic strategy to consider in those with bleeding 'downhill' varices.

Conclusion. This case serves to highlight the entity of 'downhill' varices.

Untreated hypopituitarism-causing cirrhosis in adulthood $\underline{\text { N Parsoo }},{ }^{1,2}$ V G Naidoo, ${ }^{1,2}$ K A Newton, ${ }^{1,2}$

${ }^{1}$ Inkosi Albert Luthuli Central Hospital, University of KwaZulu-Natal, Durban, South Africa

${ }^{2}$ Nelson R Mandela School of Medicine, University of KwaZulu-Natal, Durban, South Africa

Background. Hypopituitarism is a rare cause of liver disease. It results in varying degrees of cholestasis, which may progress to cirrhosis.

Case presentation. A 24-year-old woman with short stature was referred for evaluation of abnormal liver function tests. She was being investigated for hypopituitarism. Her only complaint was that of amenorrhoea. Her developmental history included obstructed labour and non-development of secondary sexual characteristics. Examination revealed jaundice, proportional short stature with no dysmorphic features. She had absent secondary sexual characteristics and no signs of chronic liver disease. No splenomegaly or ascites were found. Right upper limb Erb's palsy was present. Biochemistry showed a cholestatic hepatitis. Investigations for the aetiology of the liver disease were negative. Pituitary profile documented reduced growth hormone, cortisol, and sex hormone levels. She had a normal 46, XX karyotype. A hypoplastic pituitary was confirmed on magnetic resonance imaging. Endoscopic cholangiography revealed a non-obstructed biliary system with a paucity of intrahepatic ducts and no features of sclerosing cholangitis. Endoscopy confirmed oesophageal varices and portal hypertensive gastropathy. Liver biopsy showed cirrhosis. A diagnosis of hypopituitary-associated cirrhosis was made.

Discussion. Hypopituitarism may be accompanied by progressive cholestasis and cirrhosis if hormone replacement is delayed. Hormone deficiency may delay maturation of biliary transport mechanisms leading to bile accumulation, cholestasis with hepatitis and eventually cirrhosis.

Conclusion. It is important for paediatricians, endocrinologists and gastroenterologists to be aware of this association, as early initiation of hormone replacement may prevent patients from developing cirrhosis. 


\section{Successful pregnancy on anti-TNF- $\alpha$ therapy JH Van Zyl, WM Simmonds}

University Of The Free State, Bloemfontein, South Africa

Background. Female patients with active inflammatory bowel disease (IBD) often have difficulties with fertility. They also have great concerns regarding the safety of the drugs that are being used to treat their disease during pregnancy. Meta-analysis of the drugs used for the treatment of IBD show that none of these drugs are regarded as being absolutely safe in pregnancy. Anti-tumour necrosis factor (TNF) a therapy has been shown to be effective in inducing remission. Case reports are being published on successful pregnancies on anti-TNF- $\alpha$ therapy.

Case report. A 30-year-old women presented to us during 2008 with a history of fistulating Crohn's disease since 2004. Therapy at that stage consisted of $40 \mathrm{mg} / \mathrm{d}$ corticosteroids and $150 \mathrm{mg} / \mathrm{d}$ azathioprine. Metronidazole was stopped a year previously. On examination it was found that she had active disease with a rectal fistula and a Crohn's disease Activity Index (CDAI) of 184. Metronidazole was added to her therapy but in 2 months the CDAI only dropped to 132 . The family also paid a visit to the fertility clinic because of concerns regarding pregnancy and fertility. Anti-TNF- $\alpha$ therapy was started with infliximab (IFX) at a dose of $5 \mathrm{mg} / \mathrm{kg}$ in combination with $100 \mathrm{mg} / \mathrm{d}$ azathioprine. Corticosteroids were weaned and stopped. The patient's CDAI dropped to 94 . A year after starting therapy with IFX a normal pregnancy ensued and a healthy baby boy was delivered in March 2010. Due to difficult venous access, IFX was changed to adalumimab, $40 \mathrm{mg}$ every 2 weeks. Azathioprine remained at $100 \mathrm{mg} / \mathrm{d}$. Her CDAI dropped to 52. During 2011 she became pregnant again. The pregnancy was uncomplicated and in July 2012 a healthy baby girl was delivered. The patient's CDAI at present is 29 . Discussion. Anti-TNF- $\alpha$ therapy in combination with azathioprine in this patient: (i) induced remission, (ii) normalised fertility and (iii) proved to be safe during pregnancy. 